\title{
RATIONAL ELASTICITY OF FACTORIZATIONS IN KRULL DOMAINS
}

\author{
D. D. ANDERSON, DAVID F. ANDERSON, \\ SCOTT T. CHAPMAN, AND WILLIAM W. SMITH
}

(Communicated by Louis J. Ratliff, Jr.)

\begin{abstract}
For an atomic domain $R$, we define the elasticity of $R$ as $\rho(R)=$ $\sup \left\{m / n \mid x_{1} \cdots x_{m}=y_{1} \cdots y_{n}\right.$, for $x_{i}, y_{j} \in R$ irreducibles $\}$ and let $l_{R}(x)$ and $L_{R}(x)$ denote, respectively, the inf and sup of the lengths of factorizations of a nonzero nonunit $x \in R$ into the product of irreducible elements. We answer affirmatively two rationality conjectures about factorizations. First, we show that $\rho(R)$ is rational when $R$ is a Krull domain with finite divisor class group. Secondly, we show that when $R$ is a Krull domain, the two limits $l_{R}\left(x^{n}\right) / n$ and $L_{R}\left(x^{n}\right) / n$, as $n$ goes to infinity, are positive rational numbers. These answer, respectively, conjectures of D. D. Anderson and D. F. Anderson, and D. F. Anderson and P. Pruis. (The second question has also been solved by A. Geroldinger and F. Halter-Koch.)
\end{abstract}

\section{INTRODUCTION}

If $R$ is a UFD, then any two factorizations of a nonzero nonunit of $R$ into the product of irreducible elements have the same length. Of course, this need not be true for an arbitrary atomic domain (an integral domain is atomic if each nonzero nonunit is a product of irreducible elements (atoms)). Following Zaks [15], we define an atomic domain $R$ to be a half-factorial domain (HFD) if whenever $x_{1} \cdots x_{m}=y_{1} \cdots y_{n}$ with each $x_{i}, y_{j} \in R$ irreducible, then $m=n$. In order to measure how far an atomic domain $R$ is from being a HFD, we define the elasticity of $R$ as $\rho(R)=\sup \left\{m / n \mid x_{1} \cdots x_{m}=y_{1} \cdots y_{n}\right.$, for $x_{i}, y_{j} \in$ $R$ irreducibles $\}$. Thus $1 \leq \rho(R) \leq \infty$ and $\rho(R)=1$ if and only if $R$ is a HFD. This concept was introduced by Valenza [14], who studied $\rho(R)$ for $R$ the ring of integers in an algebraic number field. In an earlier paper, Steffan [13] studied $\rho(R)$ (without this notation) for a Dedekind domain $R$ with finite divisor class group. Recently $\rho(R)$ has been studied in more detail in [1,

Received by the editors April 12, 1991 and, in revised form, May 23, 1991.

1991 Mathematics Subject Classification. Primary 13A05, 13F05, 13G05; Secondary 06F05, 20M14, 13C20, 13A15.

The second author gratefully acknowledges support received under a University of Tennessee Faculty Development Grant. The third author gratefully acknowledges support received under the John M. Bennet Fellowship at Trinity University and the support of The University of North Carolina at Chapel Hill. The fourth author acknowledges the hospitality of Trinity University where a portion of this work was completed. 
5]. In [1, Theorem 3.2] it is shown that for any real number $r \geq 1$ or $r=\infty$, there is a Dedekind domain $R$ with torsion divisor class group $\mathrm{Cl}(R)$ such that $\rho(R)=r$. Moreover, if $r$ is rational, then $R$ may be chosen so that $\mathrm{Cl}(R)$ is finite (this is also proved in [5]). This motivated the following question in [1]: if $R$ is a Krull domain with $\mathrm{Cl}(R)$ finite, is $\rho(R)$ rational, and moreover, does $\rho(R)=m / n$, where $x_{1} \cdots x_{m}=y_{1} \cdots y_{n}$ for some irreducibles $x_{i}, y_{j} \in R$ ?

In $\S 1$ we prove a general result (Theorem 4) about when certain quotients of real-valued functions on an additive submonoid of $\mathbf{Z}_{+}^{k}$ obtain their maximum and minimum values. In the second section, Theorem 4 is then applied to answer two rationality conjectures about factorizations in Krull domains. Theorem 10 answers affirmatively the above question about the rationality of $\rho(R)$ in somewhat more generality. As an added bonus, Theorem 13 answers (part of) a conjecture of Anderson and Pruis [4] about the asymptotic behavior of length functions on integral domains; this conjecture has also recently been solved by Geroldinger and Halter-Koch [8]. The moral here is that certain finiteness conditions imply the rationality of factorization invariants.

For a nonzero nonunit $x$ in an atomic domain $R$, let

$$
\begin{aligned}
& l_{R}(x)=\inf \left\{n \mid x=x_{1} \cdots x_{n} \text { with each } x_{i} \in R \text { irreducible }\right\}, \\
& L_{R}(x)=\sup \left\{n \mid x=x_{1} \cdots x_{n} \text { with each } x_{i} \in R \text { irreducible }\right\},
\end{aligned}
$$

and

$$
\rho_{R}(x)=L_{R}(x) / l_{R}(x) .
$$

In [4] it is shown that the two limits $\bar{l}_{R}(x)$ and $\bar{L}_{R}(x)$ of $l_{R}\left(x^{n}\right) / n$ and $L_{R}\left(x^{n}\right) / n$, respectively, as $n$ goes to infinity always exist $\left(\bar{L}_{R}(x)\right.$ may be $\left.\infty\right)$. Moreover, for any $0 \leq \alpha \leq 1 \leq \beta \leq \infty$, there is an integral domain $R$ and an irreducible $x \in R$ such that $\bar{l}_{R}(x)=\alpha$ and $\bar{L}_{R}(x)=\beta$. It was also conjectured that both limits are positive rational numbers when $R$ is either a Krull domain or a Noetherian domain. In [8] this conjecture is shown to be true for Krull domains and several classes of Noetherian domains, but an example is given of a Noetherian domain $R$ with $\bar{l}_{R}(x)=0$ for an irreducible $x \in R$.

Factorization theory can be studied at several levels of generality; much of it can be abstracted to semigroups, as in [8, 9, 11, 12]. As our main interest is for integral domains, we will take the middle of the road and work with semigroups only when the added generality substantially simplifies the problem. Throughout, $R$ will always be an integral domain with group of units $U(R)$ and nonzero elements $R^{*}$. Our notation and definitions will follow [7] for Krull domains and [10] for semigroups. For $R$ a Krull domain, $X^{(1)}(R)$ is its set of height-one prime ideals and $\operatorname{Div}(R)$ is its group of divisorial ideals under the $v$-operation. $\operatorname{Div}(R)$ is free abelian on $X^{(1)}(R)$. For an additive submonoid $\Gamma$ of a free abelian monoid $\bigoplus \mathbf{Z}_{+}$, $\leq$denotes the usual product order. As usual, $\infty / a=a / 0=\infty / 0=\infty$ for any real number $a>0$. For a study of several factorization properties weaker than unique factorization in an arbitrary integral domain, see [2].

\section{MAX AND MINS}

In this section, $\Gamma$ will be a commutative, cancellative monoid, written additively, with $\Gamma^{*}$ its subsemigroup of noninvertible elements (thus $\Gamma^{*}=\Gamma \backslash\{0\}$ when $\Gamma$ is an additive submonoid of a free abelian monoid). A function 
$f: \Gamma \rightarrow \mathbf{R}_{+}$is a semilength function on $\Gamma$ if (1) $f(x+y)=f(x)+f(y)$ for all $x, y \in \Gamma$ and (2) $f(x)>0$ for all $x \in \Gamma^{*}$. Given two semilength functions $f$ and $g$ on $\Gamma$, define $\phi(f, g)=\phi: \Gamma^{*} \rightarrow \mathbf{R}_{+}$by $\phi(x)=f(x) / g(x)$ for all $x \in \Gamma^{*}$. Note that $\phi(k x)=\phi(x)$ for all integers $k \geq 1$. We define $L(\Gamma, \phi)=\sup \left\{\phi(x) \mid x \in \Gamma^{*}\right\}$ and $l(\Gamma, \phi)=\inf \left\{\phi(x) \mid x \in \Gamma^{*}\right\}$. Then $0<L(\Gamma, \phi) \leq \infty$ and $0 \leq l(\Gamma, \phi)<\infty$. Note that if $\bar{\phi}=\phi(g, f)$, then $\bar{\phi}=1 / \phi$ and $l(\Gamma, \phi)=1 / L(\Gamma, \bar{\phi})$. We next give three elementary lemmas.

Lemma 1. Let $a, b, c$, and $d$ be positive real numbers. Then $a / c<$ $(a+b) /(c+d)$ if and only if $(a+b) /(c+d)<b / d$.

Proof. $a / c<(a+b) /(c+d) \Leftrightarrow a c+a d<a c+b c \Leftrightarrow a d<b c \Leftrightarrow a d+b d<$ $b c+b d \Leftrightarrow(a+b) /(c+d)<b / d$.

Lemma 2. Let $f$ and $g$ be semilength functions on $\Gamma$ and $\phi=\phi(f, g)$. Let $x, y \in \Gamma^{*}$ such that $y-x \in \Gamma^{*}$. If $\phi(x)<\phi(y)$ then $\phi(y)<\phi(y-x)$.

Proof. Because $f(x) / g(x)=\phi(x)<\phi(y)=f(y) / g(y)=[f(x)+f(y-x)] /$ $[g(x)+g(y-x)]$, we have $\phi(y)=f(y) / g(y)<f(y-x) / g(y-x)=\phi(y-x)$ by Lemma 1 .

Lemma 3. Let $\Gamma$ be an additive submonoid of a free abelian monoid and $\phi$ : $\Gamma^{*} \rightarrow G$ be any function into a totally ordered abelian group $G$. Then there is a function $\psi: \Gamma^{*} \rightarrow \Gamma^{*}$ such that $(1) \psi(x) \leq x$ for all $x \in \Gamma^{*}$ and (2) if $x, y \in \Gamma^{*}$ with $x \leq y$ then $\phi(x) \leq \phi(\psi(y))$. (Note that $\psi$ need not be uniquely determined.)

Proof. For $x \in \Gamma^{*}, \hat{x}=\left\{z \in \Gamma^{*} \mid z \leq x\right\}$ is finite. Thus we may choose $\psi(x)=w \in \hat{x}$ with $\phi(w)$ maximum, i.e., $\phi(z) \leq \phi(w)$ for all $z \in \hat{x}$. Clearly (1) and (2) hold.

For our main theorem, we specialize to $\Gamma$ an additive submonoid of $\mathbf{Z}_{+}^{k}$ for some integer $k \geq 1$ and $\phi=\phi(f, g)$.

Theorem 4. Let $\Gamma$ be an additive submonoid of $\mathbf{Z}_{+}^{k}, f$ and $g$ be semilength functions on $\Gamma$, and $\phi=\phi(f, g)$. If $y-x \in \Gamma$ for all $x, y \in \Gamma$ with $x \leq$ $y$, then $L(\Gamma, \phi)=\phi\left(x_{0}\right)$ and $l(\Gamma, \phi)=\phi\left(y_{0}\right)$ for some $x_{0}, y_{0} \in \Gamma^{*}$. Thus $L(\Gamma, \phi)$ and $l(\Gamma, \phi)$ are each positive real numbers. If $f$ and $g$ are each rational-valued, then $L(\Gamma, \phi)$ and $l(\Gamma, \phi)$ are each positive rational numbers.

Proof. We prove the theorem for $L(\Gamma, \phi)$. Since $l(\Gamma, \phi)=1 / L(\Gamma, 1 / \phi)$, the result follows for $l(\Gamma, \phi)$ as well. Let $\psi$ be the function from Lemma 3 applied to $\phi$. Suppose that $\phi(x)<L(\Gamma, \phi)$ for all $x \in \Gamma^{*}$. Choose $y_{1} \in \Gamma^{*}$, and let $x_{1}=\psi\left(y_{1}\right)$. Suppose that $y_{1}, \ldots, y_{n} \in \Gamma^{*}$ have been chosen so that the $x_{i}=\psi\left(y_{i}\right)$ 's are incomparable and $\phi\left(x_{1}\right)<\cdots<\phi\left(x_{n}\right)$. Pick $y_{n+1} \in \Gamma^{*}$ with $\phi\left(y_{n+1}\right)>\phi\left(x_{n}\right)$, and let $x_{n+1}=\psi\left(y_{n+1}\right)$. We claim that $x_{n+1}$ is not comparable to any $x_{1}, \ldots, x_{n}$. If $x_{n+1}<x_{i}$ then $x_{n+1}<x_{i}=\psi\left(y_{i}\right) \leq y_{i}$. Hence $\phi\left(x_{n+1}\right) \leq \phi\left(\psi\left(y_{i}\right)\right)=\phi\left(x_{i}\right)$ by Lemma 3, a contradiction. On the other hand, if $x_{i}<x_{n+1}$ then $x_{n+1}-x_{i} \in \Gamma^{*}$ and $\phi\left(x_{i}\right)<\phi\left(x_{n+1}\right)$. Thus $\phi\left(x_{n+1}\right)<$ $\phi\left(x_{n+1}-x_{i}\right)$ by Lemma 2 . However, $0<x_{n+1}-x_{i}<x_{n+1}=\psi\left(y_{n+1}\right) \leq y_{n+1}$; so $\phi\left(x_{n+1}-x_{i}\right) \leq \phi\left(\psi\left(y_{n+1}\right)\right)=\phi\left(x_{n+1}\right)$ by Lemma 3 again, a contradiction. Hence we can construct an infinite set $\left\{x_{n}\right\}$ of incomparable elements in $\Gamma \subset \mathbf{Z}_{+}^{k}$, a contradiction [6, Theorem 9.18]. 
Remark 5. (a) We first show that Theorem 4 may fail if we do not assume that $y-x \in \Gamma$ when $x, y \in \Gamma$ with $x \leq y$. Let $a, b \in[0, \infty]$ with $0 \leq a<b \leq \infty$. Then $\Gamma=\left\{(m, n) \in \mathbf{Z}_{+}^{2} \mid m, n \geq 1\right.$ and $\left.a<m / n<b\right\} \cup\{(0,0)\}$ is an additive submonoid of $\mathbf{Z}_{+}^{2}$ and $f, g: \Gamma \longrightarrow \mathbf{R}_{+}$given by $f(m, n)=m$ and $g(m, n)=n$ are semilength functions on $\Gamma$. For $\phi=\phi(f, g)$, we have $l(\Gamma, \phi)=a$ and $L(\Gamma, \phi)=b$; however, $a<\phi(m, n)=m / n<b$ for all $(m, n) \in \Gamma^{*}$. Note that there are integers $m, n \geq 1$ with $(m+1, n)$, $(m, n) \in \Gamma$, but $(m+1, n)-(m, n)=(1,0) \notin \Gamma$.

(b) In Theorem 4 , it is also necessary to assume that $\Gamma$ is an additive submonoid of a finitely generated free abelian monoid, rather than of just a free abelian monoid (cf. the proof of [1, Theorem 3.2]).

\section{Applications}

In this section, we apply Theorem 4 to two rationality conjectures in factorization theory. Here, $H$ will always be a commutative, cancellative monoid, written multiplicatively, with group of units $U(H)$. We say that $H$ is reduced if $U(H)=\{1\}$. As in [12] a monoid $H$ is atomic if each nonunit of $H$ is a product of irreducible elements. For an atomic monoid $H$, we define the elasticity of $H$ as $\rho(H)=\sup \left\{m / n \mid x_{1} \cdots x_{m}=y_{1} \cdots y_{n}\right.$, for $x_{i}, y_{j} \in H$ irreducibles $\}$. Then $1 \leq \rho(H) \leq \infty$. Many of the results of $[1,5]$ on $\rho(R)$, for $R$ an atomic domain, also apply to $\rho(H)$, for $H$ an atomic monoid. In particular, if $f: H \rightarrow \mathbf{R}_{+}$is a semilength function on $H$, then $\rho(H) \leq M^{*} / m^{*}$, where $M^{*}=\sup \{f(x) \mid x \in H$ is irreducible, but not prime $\}, m^{*}=\inf \{f(x) \mid x \in$ $H$ is irreducible, but not prime $\}$, and $M^{*}=m^{*}=1$ if all irreducible elements of $H$ are prime (cf. [1, Theorem 2.1]).

Let $R$ be an integral domain. Then $H_{1}=R^{*}, H_{2}=R^{*} / U(R)$, and $H_{3}=$ $\operatorname{Prin}(R)_{+}=\left\{x R \mid x \in R^{*}\right\}$ are all multiplicative monoids and $H_{2} \approx H_{3}$. Clearly $R$ is atomic $\Leftrightarrow H_{1}$ is atomic $\Leftrightarrow H_{2}$ is atomic $\Leftrightarrow H_{3}$ is atomic. Moreover, if $R$ is atomic, then $\rho(R)=\rho\left(H_{1}\right)=\rho\left(H_{2}\right)=\rho\left(H_{3}\right)$. More generally, if $H$ is any monoid, then $H$ is atomic if and only if the reduced monoid $\bar{H}=H / U(H)$ is atomic, and in this case, $\rho(\bar{H})=\rho(H)$. One can sometimes calculate $\rho(H)$ by relating $H$ to a simpler atomic monoid. Our next result, whose simple proof will be omitted, is sufficient for our purposes.

Lemma 6. Let $\partial: H_{1} \rightarrow H_{2}$ be a homomorphism of atomic monoids.

(a) If $\partial(x)$ is irreducible whenever $x \in H_{1}$ is irreducible, then $\rho\left(H_{1}\right) \leq$ $\rho\left(H_{2}\right)$.

(b) If in addition $H_{2} \subset H_{1}$ and $\partial(x)=x$ for each $x \in H_{2}$, then $\rho\left(H_{1}\right)=$ $\rho\left(H_{2}\right)$.

Our next theorem, along with Theorem 12, are the two applications of Theorem 4.

Theorem 7. Let $H$ be an atomic monoid with only a finite number of nonassociate irreducible elements. Then $\rho(H)$ is rational. Moreover, $\rho(H)=m / n$, where $x_{1} \cdots x_{m}=y_{1} \cdots y_{n}$ for some irreducibles $x_{i}, y_{j} \in H$.

Proof. We may assume that $H$ is reduced and $z_{1}, \ldots, z_{k}$ are the irreducible elements of $H$. Then $\Gamma=\left\{\left(a_{1}, \ldots, a_{k}, b_{1}, \ldots, b_{k}\right) \in \mathbf{Z}_{+}^{2 k} \mid z_{1}^{a_{1}} \cdots z_{k}^{a_{k}}=\right.$ 
$\left.z_{1}^{b_{1}} \cdots z_{k}^{b_{k}}\right\}$ is an additive submonoid of $\mathbf{Z}_{+}^{2 k}$, and $f, g: \Gamma \rightarrow \mathbf{R}_{+}$given by $f\left(a_{1}, \ldots, b_{k}\right)=a_{1}+\cdots+a_{k}$ and $g\left(a_{1}, \ldots, b_{k}\right)=b_{1}+\cdots+b_{k}$ are semilength functions on $\Gamma$. Clearly $\Gamma$ and $\phi=\phi(f, g)$ satisfy the hypotheses of Theorem 4 and $\rho(H)=L(\Gamma, \phi)$. Thus, $\rho(H)=\phi\left(x_{0}\right)$ for some $x_{0} \in \Gamma^{*}$, and the theorem follows.

We next apply Theorem 7 to $\rho(R)$, for $R$ an atomic domain. As an immediate corollary of Theorem 7, we have that $\rho(R)$ is rational when $R$ has only a finite number of (nonassociate) irreducible elements. Such integral domains are called Cohen-Kaplansky (CK) domains and have recently been studied extensively in [3]. We state this as (cf. [1, Corollary 2.13])

Corollary 8. Let $R$ be a $C K$ domain. Then $\rho(R)$ is rational. Moreover, $\rho(R)=m / n$, where $x_{1} \cdots x_{m}=y_{1} \cdots y_{n}$ for some irreducibles $x_{i}, y_{j} \in R$.

In order to prove similar results for more general atomic domains, certain finiteness conditions are needed. For the Krull domain case, we will need

Lemma 9. Let $R$ be a Krull domain and $P_{1}, \cdots, P_{n} \in X^{(1)}(R)$. Then $\{x \in$ $R^{*} \mid x R=\left(P_{1}^{a_{1}} \ldots P_{n}^{a_{n}}\right)_{v}$ for integers $\left.a_{i} \geq 0\right\}$ has only a finite number of nonassociate irreducible elements.

Proof. Let $\Gamma=\left\{\left(a_{1}, \ldots, a_{n}\right) \in \mathbf{Z}_{+}^{n} \mid\left(P_{1}^{a_{1}} \ldots P_{n}^{a_{n}}\right)_{v}\right.$ is principal $\}$. If $x R=$ $\left(P_{1}^{a_{1}} \ldots P_{n}^{a_{n}}\right)_{v}$ for $\left(a_{1}, \ldots, a_{n}\right) \in \Gamma$, then $x$ is irreducible if $\left(a_{1}, \ldots, a_{n}\right)$ is minimal in $\Gamma$. Since $\Gamma$ has only a finite number of minimal elements, the result follows.

We can now prove the conjecture on the rationality of $\rho(R)$ stated in the introduction.

Theorem 10. Let $R$ be a Krull domain such that only a finite number of divisor classes contain prime ideals. Then $\rho(R)$ is rational. Moreover, $\rho(R)=m / n$, where $x_{1} \cdots x_{m}=y_{1} \cdots y_{n}$ for some irreducibles $x_{i}, y_{j} \in R$. In particular, this is the case if $\mathrm{Cl}(R)$ is finite.

Proof. Suppose that $\left\{[P] \mid P \in X^{(1)}(R)\right\} \subset \mathrm{Cl}(R)$ is finite. Pick $P_{1}, \cdots, P_{n} \in$ $X^{(1)}(R)$ such that for any $Q \in X^{(1)}(R),[Q]=\left[P_{i}\right]$ for a unique integer $1 \leq i \leq n$. Let $H$ be the subgroup of $\operatorname{Div}(R)$ generated by $P_{1}, \ldots, P_{n}$. Define $\alpha: \operatorname{Div}(R) \rightarrow H$ by defining it on the basis elements by $\alpha(Q)=P_{i}$ if $[Q]=\left[P_{i}\right]$. Note that $\alpha$ restricts to a homomorphism from $\operatorname{Prin}(R)_{+}$to $H \cap \operatorname{Prin}(R)_{+}$. Moreover, $\alpha(x R)$ is irreducible if and only if $x R$ is irreducible. Thus $\rho\left(\operatorname{Prin}(R)_{+}\right)=\rho\left(H \cap \operatorname{Prin}(R)_{+}\right)$by Lemma 6(b). By Lemma 9, $H \cap \operatorname{Prin}(R)_{+}$has only a finite number of irreducible elements. Hence by Theorem 7, $\rho(R)=\rho\left(\operatorname{Prin}(R)_{+}\right)=\rho\left(H \cap \operatorname{Prin}(R)_{+}\right)$is rational and $\rho(R)=\rho_{R}(x)$ for some nonunit $x \in R^{*}$.

Remark 11. (a) Since $\left\{[P] \mid P \in X^{(1)}(R)\right\}$ generates $\mathrm{Cl}(R)$, the hypotheses in Theorem 10 force $\mathrm{Cl}(R)$ to be finitely generated, and thus to be finite if $\mathrm{Cl}(R)$ is torsion.

(b) If $\left\{[P] \mid P \in X^{(1)}(R)\right\}$ is infinite, $\rho(R)$ need not be rational or even finite. Even if $\rho(R)$ is rational, we may have $\rho_{R}(x)<\rho(R)$ for each nonunit $x \in R^{*}$ (cf. the remarks after [1, Theorem 3.2]).

We next apply Theorem 4 to the asymptotic behavior of length functions. Let $H$ be an atomic monoid and $x \in H$ a nonunit. As in [4, 8], we de- 
fine $l_{H}(x)=\inf \left\{n \mid x=x_{1} \cdots x_{n}\right.$ with $x_{i} \in H$ irreducible $\}$ and $L_{H}(x)=$ $\sup \left\{n \mid x=x_{1} \cdots x_{n}\right.$ with $x_{i} \in H$ irreducible $\}$. We then define

$$
\bar{l}_{H}(x)=\lim _{n \rightarrow \infty} l_{H}\left(x^{n}\right) / n \text { and } \quad \bar{L}_{H}(x)=\lim _{n \rightarrow \infty} L_{H}\left(x^{n}\right) / n
$$

Both limits always exist $\left(\bar{L}_{H}(x)\right.$ may be $\left.\infty\right)$, and furthermore, $\bar{l}_{H}(x)=$ $\inf \left\{m / n \mid x^{n}=x_{1} \cdots x_{m}\right.$ with $x_{i} \in H$ irreducible $\}$ and $\bar{L}_{H}(x)=\sup \{m / n \mid$ $x^{n}=x_{1} \cdots x_{m}$ with $x_{i} \in H$ irreducible [4, Theorem 3; 8, Theorem 1]. We can now use Theorem 4 to give a very elementary proof of the key result of Geroldinger and Halter-Koch [8, Theorem 2].

Theorem 12. Let $H$ be an atomic monoid and $x \in H$ a nonunit such that $\left\{y \in H|y| x^{n}\right.$ for some integer $\left.n \geq 1\right\}$ has only a finite number of nonassociate irreducible elements. Then $\bar{l}_{H}(x)$ and $\bar{L}_{H}(x)$ are each positive rational numbers. Moreover, there are integers $m, n \geq 1$ so that $\bar{l}_{H}(x)=l_{H}\left(x^{k m}\right) / k m$ and $\bar{L}_{H}(x)=L_{H}\left(x^{k n}\right) / k n$ for all integers $k \geq 1$.

Proof. We may assume that $H$ is reduced and that $z_{1}, \ldots, z_{r}$ are the irreducible divisors of powers of $x$. Then $\Gamma=\left\{\left(n_{1}, \ldots, n_{r}\right) \in \mathbf{Z}_{+}^{r} \mid z_{1}^{n_{1}} \cdots z_{r}^{n_{r}}=\right.$ $x^{k}$ for some integer $\left.k \geq 0\right\}$ is an additive submonoid of $\mathbf{Z}_{+}^{r}$, and $f, g$ : $\Gamma \rightarrow \mathbf{R}_{+}$given by $f\left(n_{1}, \ldots, n_{r}\right)=n_{1}+\cdots+n_{r}$ and $g\left(n_{1}, \ldots, n_{r}\right)=k$ if $z_{1}^{n_{1}} \cdots z_{r}^{n_{r}}=x^{k}$ are semilength functions on $\Gamma$. Clearly $\Gamma$ and $\phi=\phi(f, g)$ satisfy the hypotheses of Theorem 4 , and $\bar{L}_{H}(x)=L(\Gamma, \phi)$ and $\bar{l}_{H}(x)=l(\Gamma, \phi)$. Hence $L(\Gamma, \phi)=\phi\left(x_{0}\right)$ and $l(\Gamma, \phi)=\phi\left(y_{0}\right)$ for some $x_{0}, y_{0} \in \Gamma^{*}$. Note that for any $y \in \Gamma^{*}, \phi(y)=\phi(k y)$ for all integers $k \geq 1$. The result is now clear.

By Lemma 9, the hypotheses of Theorem 12 hold for $H=R^{*}$ when $R$ is a Krull domain, and in this case, $\bar{l}_{R}(x)=\bar{l}_{H}(x)$ and $\bar{L}_{R}(x)=\bar{L}_{H}(x)$ for each nonunit $x \in R^{*}$. Thus, as a corollary, we obtain the following result of Geroldinger and Halter-Koch [8, Theorem 3], which answers part of the conjecture of Anderson and Pruis [4]. (Note that in both Theorems 12 and 13 we may of course take $m=n$.)

Theorem 13. Let $R$ be a Krull domain and $x \in R^{*}$ a nonunit. Then $\bar{l}_{R}(x)$ and $\bar{L}_{R}(x)$ are each positive rational numbers. Moreover, there are integers $m, n \geq 1$ so that $\bar{l}_{R}(x)=l_{R}\left(x^{k m}\right) / k m$ and $\bar{L}_{R}(x)=L_{R}\left(x^{k n}\right) / k n$ for all integers $k \geq 1$.

We end with an observation about the asymptotic behavior of the $\rho_{R}$ function. For any atomic domain $R$ and $x \in R^{*}$ a nonunit, we define $\bar{\rho}_{R}(x)=$ $\lim _{n \rightarrow \infty} \rho_{R}\left(x^{n}\right) \quad\left(=\bar{L}_{R}(x) / \bar{l}_{R}(x)=\sup \left\{\rho_{R}\left(x^{n}\right) \mid n \geq 1\right.\right.$ an integer $\left.\}\right)$. By [4], this limit always exists and $1 \leq \bar{\rho}_{R}(x) \leq \infty$. Moreover, for any $\gamma \in[1, \infty]$, there is an integral domain $R$ and an irreducible $x \in R$ with $\bar{\rho}_{R}(x)=\gamma$. If $R$ is a Krull domain, then by Theorem 13, $\bar{\rho}_{R}(x)$ is a positive rational number, and moreover, there is an integer $n \geq 1$ such that $\bar{\rho}_{R}(x)=L_{R}\left(x^{k n}\right) / l_{R}\left(x^{k n}\right)$ for all integers $k \geq 1$. 


\section{REFERENCES}

1. D. D. Anderson and D. F. Anderson, Elasticity of factorizations in integral domains, J. Pure Appl. Algebra (to appear).

2. D. D. Anderson, D. F. Anderson, and M. Zafrullah, Factorization in integral domains, J. Pure Appl. Algebra 69 (1990), 1-19.

3. D. D. Anderson and J. L. Mott, Cohen-Kaplansky domains: integral domains with a finite number of irreducible elements, J. Algebra (to appear).

4. D. F. Anderson and P. Pruis, Length functions on integral domains, Proc. Amer. Math. Soc. 113 (1991), 933-937.

5. S. Chapman and W. W. Smith, An analysis using the Zaks-Skula constant of element factorizations in Dedekind domains, submitted.

6. A. H. Clifford and G. B. Preston, The algebraic theory of semigroups, Amer. Math. Soc. Surveys, vol. II, Amer. Math. Soc., Providence, RI, 1967.

7. R. M. Fossum, The divisor class group of a Krull domain, Springer-Verlag, New York, 1973.

8. A. Geroldinger and F. Halter-Koch, On the asymptotic behavior of lengths of factorizations, J. Pure Appl. Algebra (to appear).

9. A. Geroldinger and G. Lettl, Factorization problems in semigroups, Semigroup Forum $\mathbf{4 0}$ (1990), 23-38.

10. R. Gilmer, Commutative semigroup rings, The Univ. of Chicago Press, Chicago, 1984.

11. F. Halter-Koch, Halbgruppen mit Divisorentheorie, Exposition. Math. 8 (1990), 27-66.

12. _ Finiteness theorems for factorizations, Semigroup Forum (to appear).

13. J. Steffan, Longueurs des décompositions en produits d'éléments irréductibles dans un anneau de Dedekind, J. Algebra 102 (1986), 229-236.

14. R. J. Valenza, Elasticity of factorizations in number fields, J. Number Theory 39 (1990), 212-218.

15. A. Zaks, Half-factorial domains, Bull. Amer. Math. Soc. 82 (1976), 721-724.

Department of Mathematics, The University of Iowa, Iowa City, Iowa 52242

Department of Mathematics, The University of Tennessee, Knoxville, Tennessee 379961330

Department of Mathematics, Trinity University, San Antonio, TeXas 78212

Department of Mathematics, The University of North Carolina at Chapel Hill, Chapel Hill, North Carolina 27599-3250 